\title{
Calcium - an important diagnostic tool for the first stars
}

\author{
Andreas J. Korn ${ }^{1}$ \& Lyudmila Mashonkina ${ }^{2}$ \\ ${ }^{1}$ Uppsala Astronomical Observatory (UAO), Box 515, 75120 Uppsala, Sweden \\ email: akorn@astro.uu.se \\ ${ }^{2}$ Institute of Astronomy, Russian Academy of Sciences, 48 Pyatnitskaya St., 119017 Moscow, \\ Russia \\ email: lima@inasan.ru
}

\begin{abstract}
For refining cosmochemical studies, we are investigating the statistical equilibrium (non-LTE) of neutral and singly-ionized calcium (Ca I/II) in the atmospheres of solar-type stars. The envisioned applications are $a$ ) detailed abundance studies beyond classical models, $b$ ) using $\mathrm{Ca}$ I/II as a gravity indicator for ultra-metal-poor (UMP) stars and $c$ ) detailed modelling of the Ca II IR triplet lines for the astrophysical parametrization of the Gaia mission. We present first results for some well-studied reference cases, both at solar and low metallicity.
\end{abstract}

Keywords. Radiative transfer, line: formation, methods: numerical, stars: late-type, stars: atmospheres, stars: abundances, techniques: spectroscopic, stars: fundamental parameters

\section{Introduction}

Astronomers have tried to delineate abundance trends in the Galaxy for decades. As far as the halo is concerned, a real breakthrough has been achieved in recent years: abundance trends could, for the first time, be mapped using high-quality data of large samples of metal-poor stars with $[\mathrm{Fe} / \mathrm{H}]$ down to $\approx-4$. The most astonishing finding is the absence of measurable cosmic abundance variations at a given metallicity for most elements (Cohen et al. 2004, ApJ 612, 1107; Cayrel et al. 2004, A\&A 416, 1117).

Many of the abundance trends are flat over a wide range of $[\mathrm{Fe} / \mathrm{H}]$. Others, notably $[\mathrm{Cr} / \mathrm{Fe}]$, show a well-defined sloping trend that is hard to explain with standard supernova II yields (Cayrel et al. 2004). However large or small these trends are, efforts have to be made to explain them. One first step in this direction is to ascertain that the trends are truly cosmic, rather than the effect of inappropriate modelling assumptions.

One of these assumptions, usually made in chemical abundance analyses, is the validity of local thermodynamic equilibrium (LTE) in the line formation of cool stars. Especially in the atmospheres of metal-poor stars this assumption is questionable: electrons as collisional partners are rare, the UV fluxes are high and drive photo-ionization. In this work, we test to what extent departures from LTE (non-LTE effects) alter calcium abundances in solar-type stars of various metallicities.

\section{The Modelling}

Atomic data for the model atom including photo-ionization cross-sections were taken from TOPBASE, line data from VALD. Our final model atom contains 63 bound levels of CaI, 36 bound levels of CaII and the ground state of CaIII. We treat collisional processes involving electrons using standard formulae. We also tested the influence of inelastic hydrogen collisions on the statistical equilibrium (see below). More details will be given in a forthcoming paper (Korn \& Mashonkina, A\&A, in prep.). 

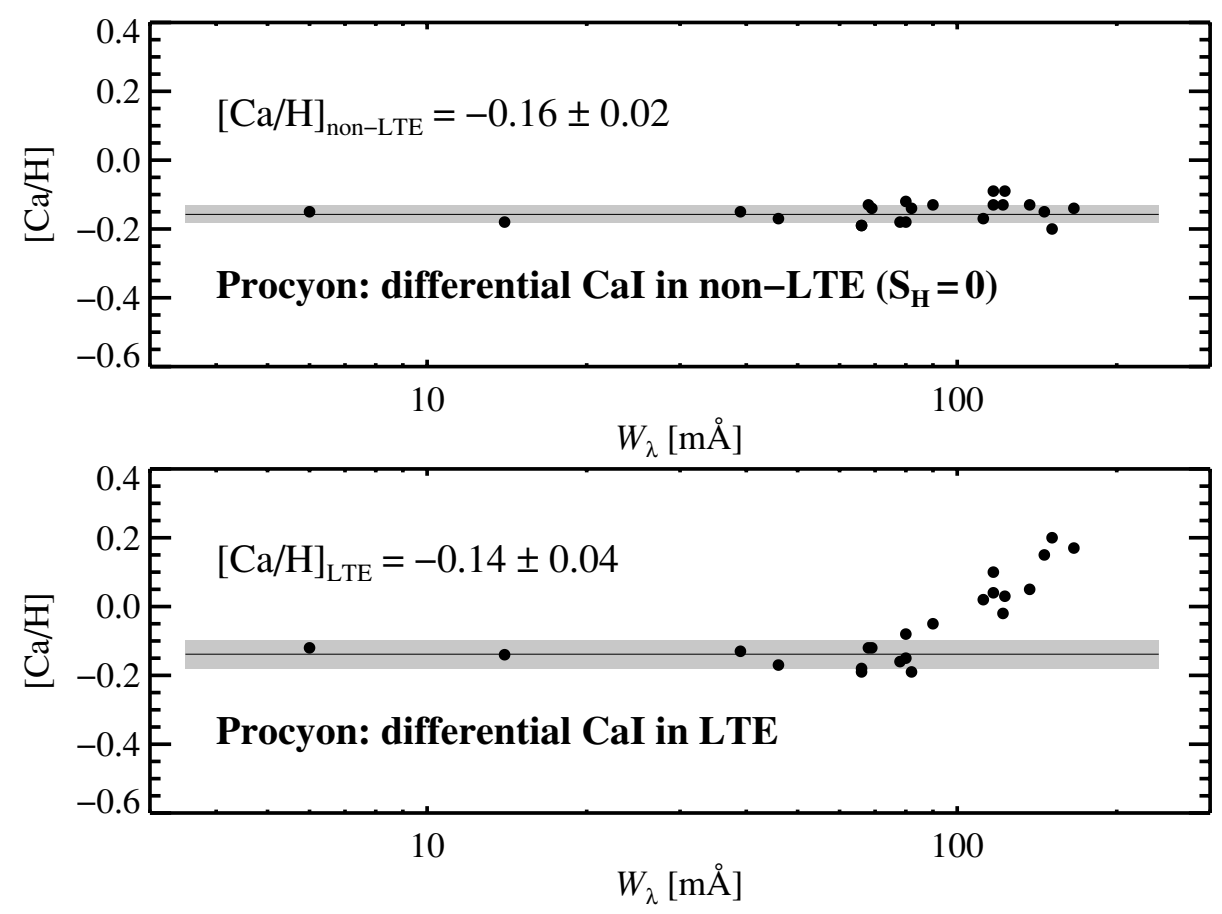

Figure 1. Differential non-LTE (top) and LTE (bottom) line abundances of Ca I lines in Procyon. Departures from LTE become increasingly important at equivalent widths above $80 \mathrm{~m} \AA$ and result in a severe trend of abundance with line strength in LTE.

\section{First Results}

We found the level populations to be rather insensitive to the assumed strength of hydrogen collisions, both at solar and low metallicity. All results presented here are computed without hydrogen collisions, as indicated by the label $\mathrm{S}_{\mathrm{H}}=0$ in Figure 1.

For the Sun, the mean non-LTE effect is $\Delta=+0.03$ dex resulting in a mean non-LTE abundance of $\log \varepsilon(\mathrm{Ca})_{\odot}=6.39 \pm 0.06$. As lines behave differently, the line-to-line scatter is somewhat reduced with respect to LTE.

Figure 1 shows $[\mathrm{Ca} / \mathrm{H}]$ in Procyon $\left(T_{\text {eff }}=6510 \mathrm{~K}, \log g=3.96,[\mathrm{Fe} / \mathrm{H}]=-0.03\right.$, Korn et al. 2003, A\&A 407, 691) as a function of equivalent width. Significant non-LTE effects are found for stronger lines and bring them into agreement with weaker ones. The mean abundances (for lines with $\mathrm{W}_{\lambda}<70 \mathrm{~m} \AA$ ) almost coincide and are surprisingly low.

In metal-deficient stars, overionization dominates the behaviour of Ca I lines leading to positive abundance corrections. For the Ca II triplet lines, the lower (upper) level is overpopulated (underpopulated) with respect to LTE significantly strengthening the lines (negative abundance corrections). For example, the mean Ca I abundance correction for the metal-poor turnoff star HD $84937\left(T_{\text {eff }}=6350 \mathrm{~K}, \log g=4.00,[\mathrm{Fe} / \mathrm{H}]=-2.16\right.$, Korn et al. 2003) is +0.11 dex, while for Ca II $8498 \AA$ it is -0.18 dex.

The Ca I resonance line at $4227 \AA$ in the UMP star HE 0107-5240 (Christlieb et al. 2002, Nature 419,904$)$ is weakened $(\Delta=+0.5 \mathrm{dex}$ !) bringing it into good agreement with Ca II $3933 \AA$ at the gravity inferred from the non-detection of optical Fe II lines.

Along with corrections from 3D hydrodynamical model atmospheres, non-LTE effects will have to be taken into account, if we ever want to achieve an abundance accuracy of 0.2 dex when analysing stars over a wide range of metallicity. 